\title{
ArcheoSciences
}

Revue d'archéométrie

$31 \mid 2007$

Varia

\section{Les prospections des ferriers de Castel-Minier : approche interdisciplinaire}

The surveys of Castel-Minier slag heaps: interdisciplinary approach

\section{Gérald Bonnamour, Nicolas Florsch et Florian Téreygeol}

\section{(2) OpenEdition}

1 Journals

Édition électronique

URL : https://journals.openedition.org/archeosciences/712

DOI : 10.4000/archeosciences.712

ISBN : 978-2-7535-1596-3

ISSN : 2104-3728

Éditeur

Presses universitaires de Rennes

Édition imprimée

Date de publication : 31 décembre 2007

Pagination : $37-44$

ISBN : 978-2-7535-0618-3

ISSN : $1960-1360$

\section{Référence électronique}

Gérald Bonnamour, Nicolas Florsch et Florian Téreygeol, « Les prospections des ferriers de CastelMinier : approche interdisciplinaire », ArcheoSciences [En ligne], 31 | 2007, mis en ligne le 31 décembre 2009, consulté le 01 février 2022. URL : http://journals.openedition.org/archeosciences/712 ; DOI : https://doi.org/10.4000/archeosciences.712 


\title{
Les prospections des ferriers de Castel-Minier : approche interdisciplinaire
}

\author{
The surveys of Castel-Minier slag heaps: interdisciplinary approach
}

\author{
Gérald Bonnamour*, Nicolas Florsch ${ }^{* *}$, Florian TéReYgeol ${ }^{* * *}$
}

\begin{abstract}
Résumé : Au XIV siècle, le site de Castel-Minier (commune d'Aulus-les-Bains, Ariège) a fait l'objet d'une importante exploitation pour le plomb et l'argent. Pendant quelques décennies, cette mine d'argent a été la plus importante du royaume de France. Ce gîte a d'ailleurs laissé de belles traces tant dans les archives que sur le terrain (Dubois, 2000). Les recherches entreprises depuis 2003 visent à mieux comprendre comment s'est faite cette exploitation (Téreygeol, 2004). Nous présentons les prospections et sondages préalables à des interventions archéologiques plus lourdes, sur la rive droite du site : topographie en maile de $0,5 \mathrm{~m}$ par $0,5 \mathrm{~m}$, prospections magnétique et électrique, ainsi qu'un ensemble de carottages pédologiques. Nous montrons comment ce mode pluridisciplinaire de prospection s’avère pertinent, puisqu'il permet de dégager clairement l'organisation du site, et notamment la présence de deux ferriers distincts, ce qui doit conduire l'archéologue à établir sa stratégie de fouille et d'interprétation en fonction de cette organisation. Le site étant multi-métallique ( $\mathrm{Ag}, \mathrm{Pb}, \mathrm{Fe})$, les investigations préparatoires à la fouille ont été plus poussées afin que la recherche sur une thématique n'en oblitère pas une autre. Enfin un sondage archéologique a permis d'avoir une idée ponctuelle de l'ensemble corroborant les données déjà acquises.

Abstract: Castel-Minier site was one of the most important silver mines in the French kingdom during the second part of the $14^{\text {th }}$ Century. New research began in 2003 and carried out two slags deposits which showed bloomery smelting process as smelting and steel production. This iron production could be bound to silver one. But archives put in light an agreement in 1347 between the community of Sem who exploited Rancie iron mine and the lord of Couserans. Men of Couserans exchanged charcoal against iron ore from Rancié. The location of two kinds of metallurgy (silver and iron) on a same site is quite rare and should be study carefully. So we decided to engage three kinds of survey (magnetic, electric and pedological). We wish to show how three ways of survey prove to be relevant. We are able to understand the organization of the site. In addition the archaeologist can clearly manage his excavation strategy. At least, an archaeological survey has been done to confirm the first conclusion we had.
\end{abstract}

Mots clés : Archéologie minière, Paléométallurgie, Prospections géophysiques, Pédologie, topographie, Castel-Minier.

Key words: Mining archaeology, Ancient metallurgy, Geophysical prospecting, Soil science, Topography, Castel-Minier.

\section{INTRODUCTION}

Le site de Castel-Minier à Aulus en Couserans (Ariège) est connu pour être une des plus importantes mines d'argent du royaume de France dans la première moitié du XIV siècle (Fig. 1). La reprise des recherches sur ce lieu en 2003 a conduit à la localisation de deux amas de scories témoignant à la fois d'une activité de forge et de transformation du minerai de fer. Cet ensemble lié intimement à l'activité minière d'extraction d'argent s'inscrit dans une problématique d'échange du charbon de bois de la vallée de CastelMinier contre le célèbre minerai de fer du Rancié de la vallée

\footnotetext{
*Arkemine-La Mine de Rozier, 63230 Saint-Pierre-le-Chastel. (accueil@arkemine.fr)

** UMR 7619, CNRS «Sisyphe»-Université Pierre et Marie Curie, Paris. (florsch@ccr.jussieu.fr)

***UMR 5060 IRAMAT-LMC 90010 Belfort, UMR 9956 - Laboratoire Pierre-Süe, CEA Saclay, 91191 Gif-sur-Yvette Cedex. (florian.tereygeol@cea.fr)
} 


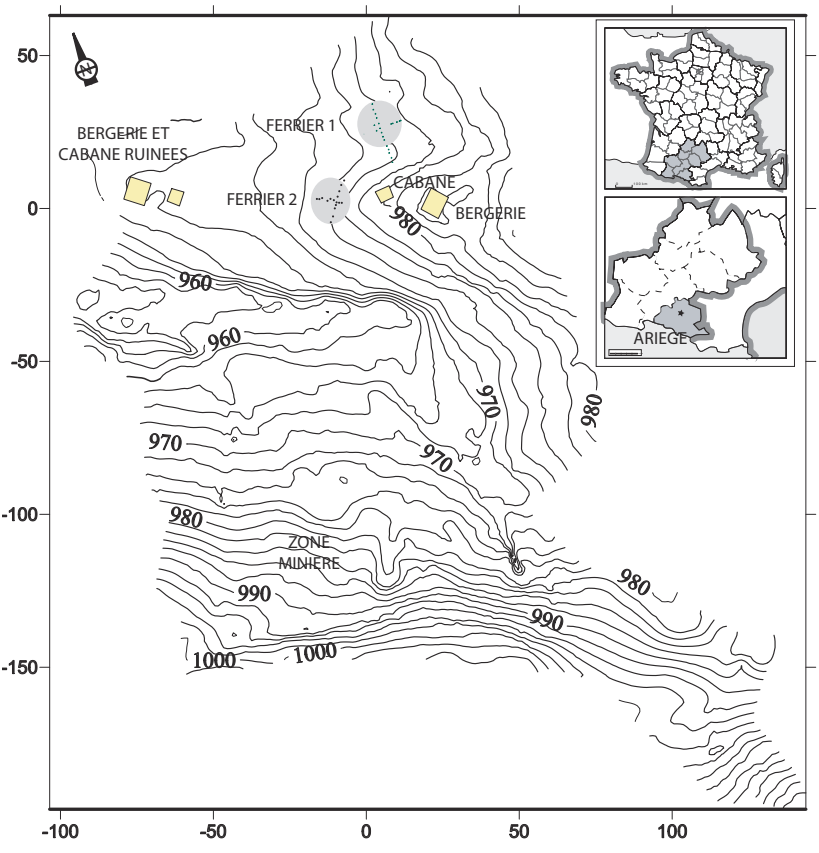

Figure 1 : Localisation et cartographie du site de Castel-Minier. Figure 1: Localization and cartography of the site.

voisine. Un accord de 1347-1348 renseigne, en effet, sur les clauses régissant ce marché qui a perduré jusqu’à la révolution. L'absence de données archéologiques sur la production du fer en vallée d'Aulus, l'association sur un même site des métallurgies du fer et des non-ferreux nous a incité à la prudence dans nos stratégies de fouille. En effet, il est classiquement admis que les vestiges de la métallurgie extractive du fer marquent plus le paysage que ceux de la métallurgie de l'argent. C'est pourquoi nous avons choisi de coupler une approche microtopographique à des prospections magnétique et électrique. Fort des cartes obtenues, des carottages ont pu être réalisés sur les zones des ferriers.

\section{LES DONNÉES GÉOPHYSIQUES}

\section{Cartographie magnétique}

Les prospections géophysiques permettent d'approcher tant stratégiquement que tactiquement les terrains archéologiques (Scholar et al., 1990, Dabas et al., 2006). La prospection magnétique, en tant qu'outil performant pour la détection des vestiges liés aux arts du feu, est particulièrement adaptée pour une première délimitation des vestiges paléométallurgiques et paléosidérurgiques. Cela résulte de la présence de magnétite dans les restes que sont les scories, culots, battitures, fours etc. Entreprise en 2003, avant les premiers sondages (et ce afin de ne pas perturber les sources magnétiques par des excavations), la cartographie magnétique met en évidence une zonation particulière pouvant participer à la bonne compréhension de l'organisation spatiale du site, au moins dans sa partie sidérurgique. En effet, les résultats obtenus révèlent des zones bien distinctes, et notamment la présence d'un second ferrier qui repose en dessous de la cabane de berger actuelle : ces éléments de répartition, bien lisibles sur la carte magnétique (Fig. 2) peuvent être pris en compte avant même de procéder aux sondages révélateurs. Ajoutons que la carte magnétique révèle également les zones où l'on acquiert, grâce à elle, la certitude qu'il n'existe pas de vestiges magnétiques. Ainsi, la prospection magnétique s'est posée, sur ce site comme sur d'autres, comme une étape évidente dans le cadre d'une recherche paléosidérurgique, en aval des premières recherches de terrain comme d'archives, mais en amont d'une logique de fouille qu'elle contribue à orienter.

En terme de mise en œuvre, la carte magnétique du site combine des mesures au magnétomètre à pompage optique à vapeur de Césium (G858-G de Geometrics, de l'UMR 7619 - Sisyphe) et des mesures au plus traditionnel magnétomètre à proton (GEM 19T de l'UMR 5060 - IRAMAT). Ce dernier instrument, plus lent à mettre en œuvre, est nécessaire dans les secteurs ayant une topographie accidentée (partie NordEst) qui oblige à des mesures point par point, tandis que le magnétomètre à Césium est du type "walking " (acquisition au rythme de la marche). Ainsi, les cartes obtenues ne sont pas homogènes: pour le "Césium ", la maille est de $0,2 \mathrm{~m}$ par $1 \mathrm{~m}$, avec les deux capteurs du gradiomètre proche du sol ( $30 \mathrm{~cm}$ et $1 \mathrm{~m})$. Au «proton", la maille est de $2 \mathrm{~m}$ par $2 \mathrm{~m}$, les capteurs situés plus en hauteur (1,5 m et $2 \mathrm{~m}$ ). Une opération d'homogénéisation est rendue possible en effectuant un prolongement vers le haut des mesures au Césium (traitement dans le domaine de Fourier, [Blakely, 1996]), qui ramènent toutes les mesures à la même hauteur, ce qui permet une lecture globale. Il est utile de noter que dans une telle recherche de structures importantes, il est préférable de mesurer le champ magnétique à une hauteur minimale, sous peine d'être saturé par les hétérogénéités superficielles.

L'interprétation de ces cartes peut se faire en deux phases, la première étant strictement géophysique, la deuxième consistant à tirer des leçons archéologiques des résultats (ce qui est présenté ultérieurement dans le texte). D’un point de vue géophysique, la carte magnétique du site met en évidence trois zones d'anomalie qui appellent quelques commentaires. Dans la partie Est prospectée à l'aide du GEM 19T, l'anomalie dipolaire (vers $\mathrm{x}=20, \mathrm{y}=40$ ) entourée d'un cercle correspond à la réponse que pourrait donner un four. En effet, l'expérience acquise ces dernières années 

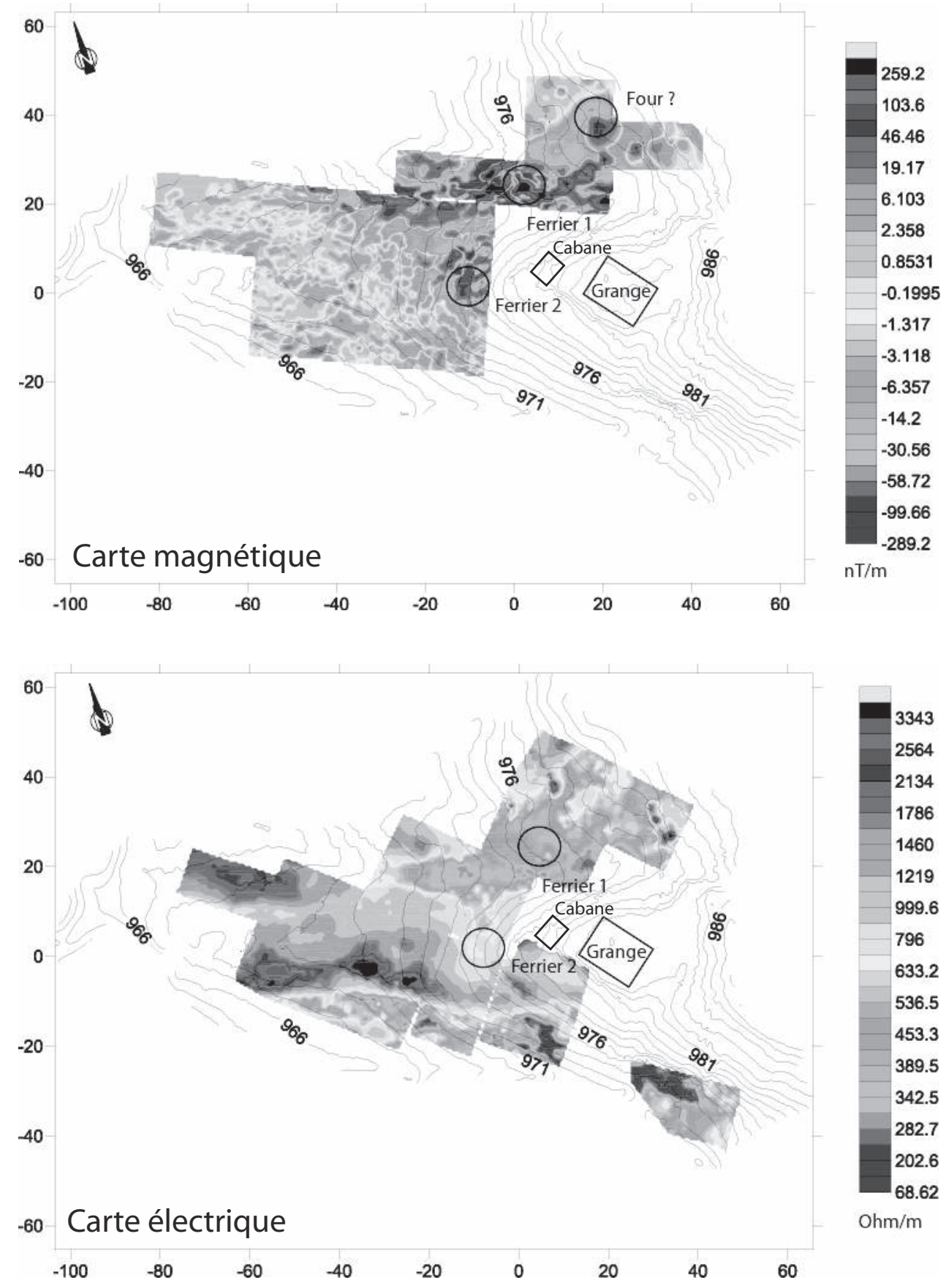

Figure 2 : Cartes magnétique et électrique.

Figure 2: Electric and magnetic maps.

conduit à considérer que, dans la plupart des cas d'atelier, les amas de scories produisent des anomalies magnétiques plus intenses et plus désorganisées que les fours (par simple effet d'accumulation, voir à ce propos Beyrie et al., 2005). En même temps, les anomalies de fours apparaissent isolées, conduisant le «standard anomalique » à être typé, avec, d'un côté, une ou plusieurs anomalies confinées, conformes à un modèle élémentaire de dipôle, et d'un autre un complexe anomalique de grande amplitude relevant de multiples sources (plus ou moins étendues). La lecture détaillée de ces dernières est rendue impossible (mais sans doute aussi, s'agissant d'amas de scories, peu utile), les anomalies se superposant de manière chaotique (ce qui n'est pas sans lien avec la manière dont les scories ont pu être déversées dans la pente). 
On observe bien, dans la partie centrale du site, en avalpente de la zone cerclée "Ferrier 1 ", un lot d'anomalies de plus ou moins forte intensité qui dessine précisément cette zone d'épandage des scories. Ces dernières sont contenues dans un chenal sur lequel nous reviendrons. Grâce à l'effet de pente qui disperse les scories vers le bas, il se dessine nettement la zone d'activité (zone centrée sur le four supposé) bien isolée de celle servant au rejet des scories dans la pente. Par ailleurs, un deuxième groupe coalescent apparait en limite de carte sous la cabane de berger. Elle semble épouser la forme du terrain et son ampleur la rapproche de ce qui a été observé dans le chenal montrant des ruptures d'orientation qui n'apparaissent pas comme des phénomènes naturels.

En fin de compte, rappelons que les cartes magnétiques ne donnent qu'une lecture partielle d'un tel site (la lecture des seules structures et vestiges magnétiques), et qu'il est pertinent de tenter de compléter cette image par celle faisant apparaitre d'autres paramètres physique, tel que la résistivité.

\section{Cartographie électrique}

La résistivité apparente, grandeur mesurée par la méthode électrique, est principalement représentative de la teneur en eau. Cette dernière peut varier pour des raisons géologiques, pédologiques, mais signe également la présence de structures en dur (murs par exemple). La cartographie électrique est traditionnelle parmi les méthodes géophysiques appliquées à l'archéologie (Scollar et al., 1990; Telford et al., 1990).

Nous avons mis en œuvre une méthode pôle-pôle, offrant le meilleur compromis entre résolution et profondeur d'investigation (Panissod et al., 1998)

Une vue d'ensemble de la carte révèle une zonation organisée par une orientation NO-SE (Fig. 2). Elle est parallèle à l'axe du torrent sous-jacent qui a déterminé l'incision torrentielle de ce fond de vallon. Cette zonation est très vraisemblablement liée aux chenaux naturels qui ont été comblés par des sédiments fins. Leurs présences se traduit par les axes conducteurs majeurs de la carte. Il y a donc une corrélation entre carte électrique et carte topographique, qui résulte de deux facteurs relatifs aux hauts topographiques (relatifs). Dans un cas, les résistants de ces hauts sont associés à la zone non saturée, plus résistante par l'absence d'eau. Dans l'autre cas, c'est la roche qui est à faible profondeur et détermine à la fois ces maxima de topographie et de résistivité.

Cependant, la méthode pôle-pôle, avec une distance interélectrode métrique, n'est pas sensible aux terrains en-dessous du mètre de profondeur. Conscient des limites que cela implique pour une interprétation paléo-environnementale, nous avons complété, par précaution, ces mesures cartographiques de " traînés " par des sondages électriques (qui ont un champ d'investigation plus profond), et par des « panneaux électriques » qui produisent des coupes verticales de résistivité. Le panneau électrique réalisé sous la cabane de berger a montré notamment qu'il existe bien un résistant en surface, mais surtout que l'on passe très rapidement, sous un mètre de terrain, à une zone conductrice qui atteste de la présence d'un remplissage saturé d'eau dans une dépression de la roche ou dans une arène bien développée. L'exploitation par ailleurs des sondages électriques atteste également ce résultat. Finalement, deux ensembles se détachent : la zone résistante représentée par le maximum qui correspond à la proximité immédiate de la roche et une zone de moindre résistance qui correspond à un sédiment grossier complètement délavé et très perméable (paléo-chenal ou structure anthropique).

En changeant d'échelle, c'est-à-dire en étant attentif aux structures de courtes longueurs d'onde (aux détails), plusieurs anomalies attirent notre attention. Elles s'inscrivent toutes dans un quadrilatère ${ }^{1}$ correspondant aux zones les plus conductrices. Toute la zone supérieure, à proximité du four supposé, semble présenter des structures significatives et organisées. Dans le reste de la carte, il est difficile de distinguer le géologique de l'anthropique. Pour le géophysicienarchéologue, la corrélation imparfaite de la carte électrique et de la carte magnétique n'est pas une surprise en soi : le contraire n'est rencontré que pour des cas particulier (vestiges en brique, gallo-romains, par exemple, ou bien, en géologie, lors de variations de lithologie). On notera pourtant l'accumulation d'indices tant magnétiques qu'électriques sur la zone autour du point $(10,0)$.

\section{LES DONNÉES PÉDOLOGIQUES}

\section{Méthode}

À ce moment de la recherche, seules des informations en deux dimensions sont disponibles concernant les ferriers. Le premier d'entre eux a bien un volume marquant le paysage mais il est difficile, pour ne pas dire impossible, de déconvoluer les courbes de niveau afin de retrouver et les formes $\mathrm{du}$ terrain naturel et celles du ferrier ${ }^{2}$. Dans le second cas, la topographie ne montre pas de différence notable et seule

1. Les angles de ce rectangle sont cotés dans notre référentiel : $-10,5$; $-20,20 ; 25,25,20,40$.

2. Les fouilles qui ont suivies ce travail, ont montré la pertinence de la remarque. En effet, il a été mis en évidence un aménagement de la pente sur laquelle le ferrier a été installée 
la prospection magnétique a pu révéler ce deuxième amas. C'est pourquoi, afin d'apprécier le volume existant des deux ferriers ainsi que l'évolution des couches, des séries de carottages ont été mis en place sur ces deux accumulations de scories. Les résultats obtenus sont couplés aux informations topographiques, magnétiques et électriques disponibles. On obtient ainsi une image très détaillée des niveaux archéologiques avant toute intervention. Les prélèvements pour la réalisation des carottages ont été faits à la tarière manuelle permettant d'atteindre 2,5 $\mathrm{m}$ de profondeur. L'espacement entre chaque carotte est métrique offrant ainsi une information bien corrélée à la surface estimée des ferriers. Cette méthode permet d'observer et d'apporter des informations liées à la stratification sans être invasif. L'ensemble des carottages a été réalisé sur des axes choisis sur le terrain afin de recouper au mieux les deux ferriers (2 axes disposés perpendiculairement l'un à l'autre pour chacun des ferriers). Ces axes ont ensuite été levés au tachéomètre pour préciser l'implantation de chaque carottage et pour comparer ces données à la microtopographie locale. Des coupes schématiques sont proposées pour chacun des axes choisis.

\section{Ferrier 1}

Sur le premier ferrier (Fig. 3), les anomalies magnétiques sont très marquées : elles évoluent entre -100 et plus de 400 $\mathrm{nT} / \mathrm{m}$. Une anomalie topographique existe également. En revanche, cette zone n'a pas une résistivité très élevée (autour de $350 \mathrm{Ohm} . \mathrm{m}$ ) du moins par rapport à son environnement immédiat. La somme des 19 carottages donne une succession stratigraphique qui peut être résumée du haut vers le bas comme suit. Il se trouve d'abord un niveau argilo limoneux d'épaisseur assez constante (de l'ordre de $50 \mathrm{~cm}$ ) de couleur brune foncée sans artefact archéologique. Il est suivi par un niveau limono-argileux brun où apparaissent des charbons et des scories éparses. La puissance de ce niveau varie de $1 \mathrm{~m}$ à une dizaine de centimètres. Immédiatement sous-jacent, se trouve le ferrier proprement dit composé de scories (coulées ou non), de charbons dans une matrice limono-argileuse. Fait notable, un liseré argileux d'un centimètre d'épaisseur scinde l'accumulation des scories en deux entités bien distinctes. Sous le ferrier, deux niveaux ont encore été perçus dans quelques sondages: le plus haut est argilo-limoneux brun clair avec des cailloutis, le plus profond est un limon sableux. Dans le cas de la série transversale de prélèvements, le passage de la carotte 5 à la carotte 4 montre la disparition brutale du ferrier. Dans l'axe longitudinal, la décroissance est plus régulière et l'absence des couches de scories dans le sondage 10 traduit d'abord l'impossibilité qu'il y a eu à carotter plus profondément. Ainsi dans sa partie nord, le ferrier décroît régulièrement alors qu'au sud son arrêt est particulièrement brutal.

\section{Ferrier 2}

Le second ferrier ne montre pas d'anomalie topographique particulière (Fig. 4). À son endroit, la résistivité du sol est plus importante (de l'ordre de $700 \mathrm{Ohm} . \mathrm{m})$. En revanche,

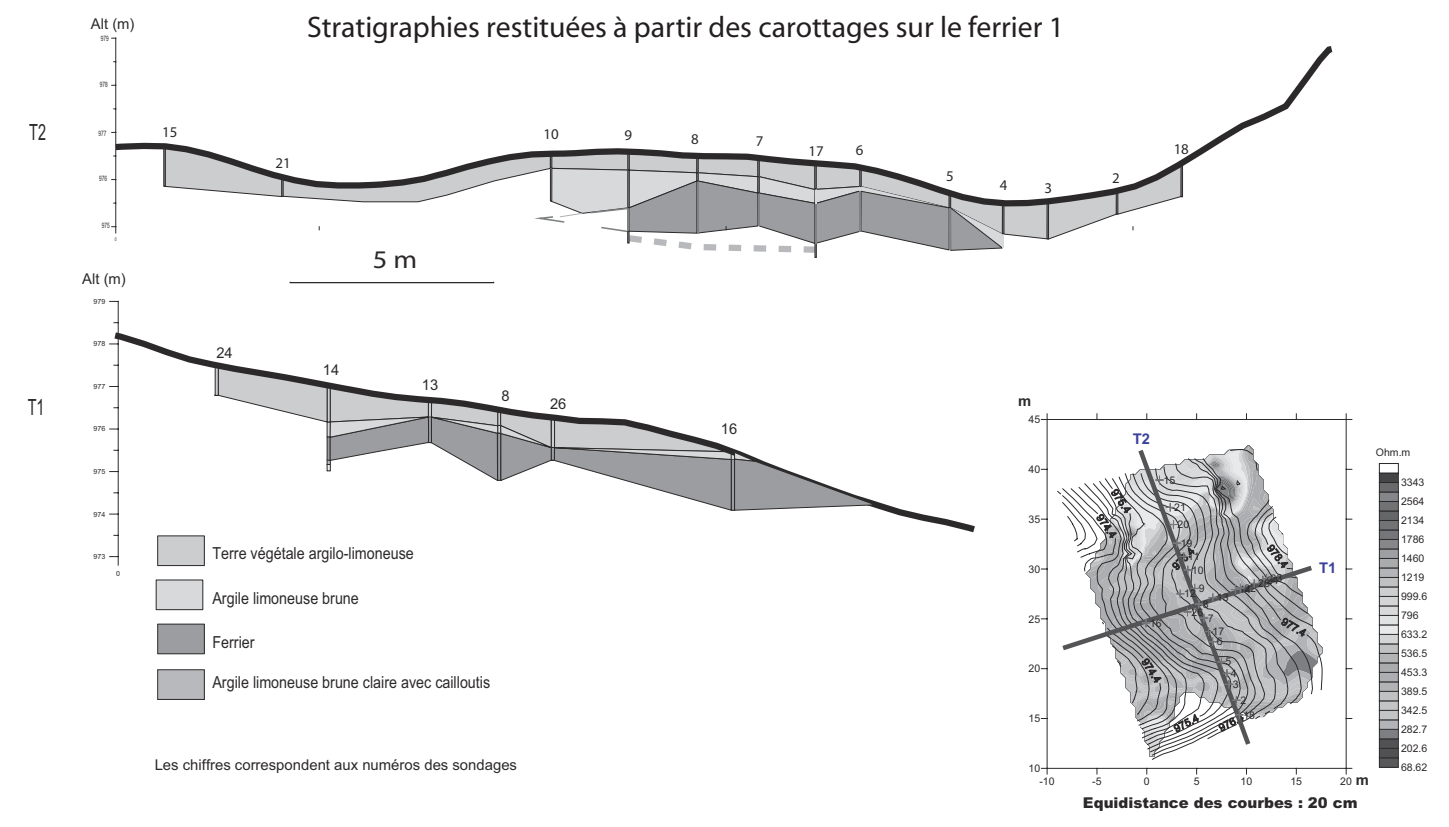

Figure 3 : Séries de carottages du ferrier 1.

Figure 3: First slag heap drilling series. 


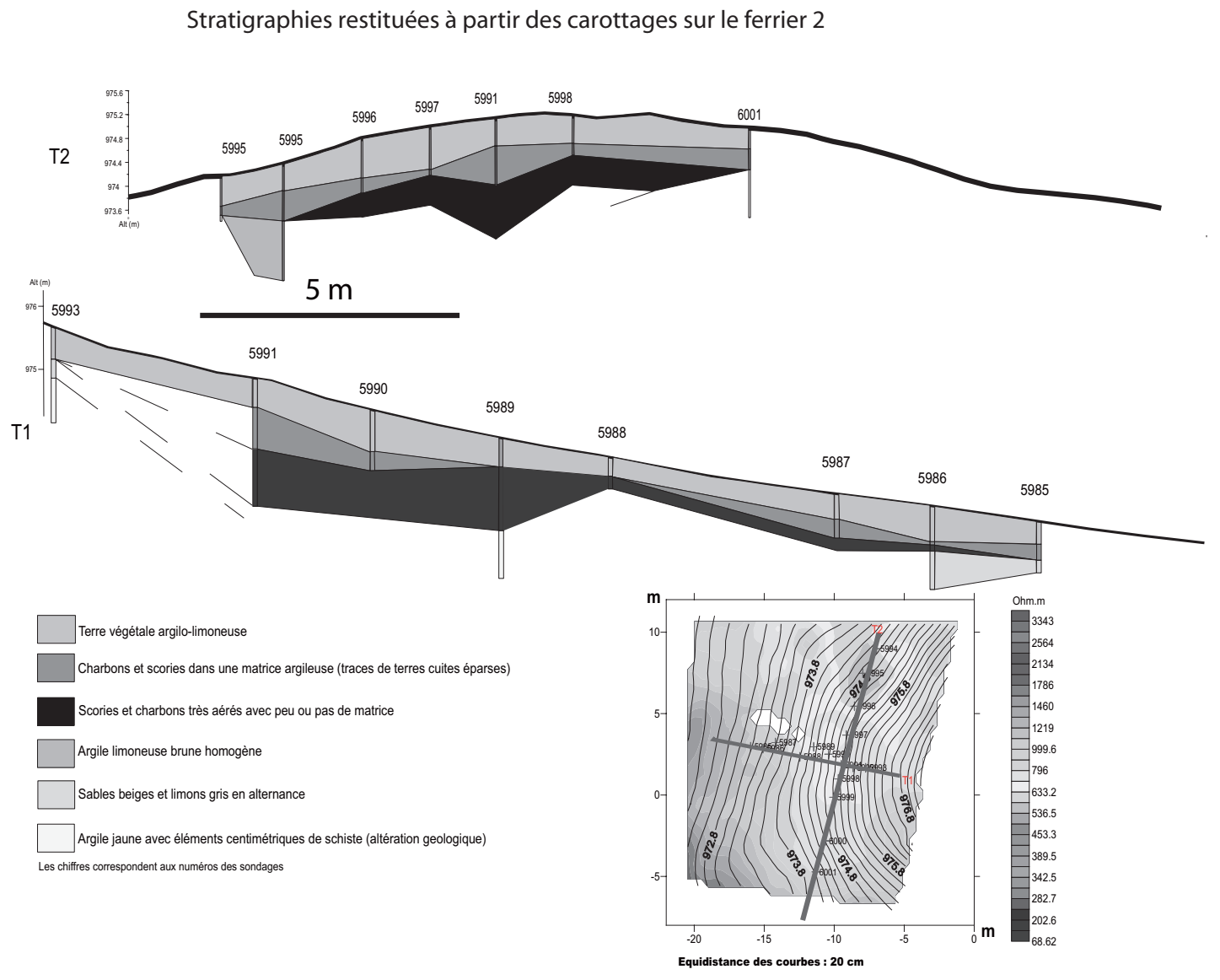

Figure 4 : Séries de carottages du ferrier 2.

Figure 4: Second slag heap drilling series.

l'image fournie par la prospection magnétique montre un alignement qui ne semble guère naturel avec des amplitudes équivalentes à celles rencontrées sur le premier ferrier. La série stratigraphique retrouvée à l'occasion des carottages se résume en six couches. On retrouve d'abord le même niveau argilo-limoneux, suivi par une strate argileuse riche en charbons avec quelques scories et des traces de terre cuite. Vient le ferrier dont les vides entre les scories n'ont pas été comblés, rendant son carottage délicat. Cet amas repose sur trois substrats différents qu'il n'est possible en l'état d'organiser stratigraphiquement. Il s'agit d'un niveau d'argile limoneuse homogène, de sables beiges bien triés alternant avec des limons grisés et un niveau argileux jaune foncé avec des éléments de schistes. Cette dernière couche pourrait être assimilée au niveau d'altération du substratum alors que les sables beiges alternant avec des limons grisés rappellent les descriptions de sables issus du traitement minéralurgique.

Le niveau de scories sans matrice est très drainant et aéré, plus résistant d'un point de vue géophysique qu'avec une matrice argileuse riche en eau. La comparaison des cartes des résistivités au niveau des deux ferriers semble confirmer cette hypothèse.

\section{Sondage ARCHÉOlOgiQUe SUR LE FERRIER 1}

Un premier sondage de vérification a été entrepris sur le ferrier 1 (Fig. 5). Outre le fait qu'il a donné lieu à la découverte d'éléments de la chaîne opératoire de production du fer particulièrement prometteurs (Dillmann et al., 2005), il a conduit à une première validation des résultats avancés par les différentes opérations de prospection. La stratigraphie retrouvée corrobore celle restituée à l'occasion des carottages. Comme à l'habitude dans ce genre de dépôt, les niveaux stratigraphiques sont très similaires les uns par rapport aux autres. Passé la couche de terre végétale, on note sur près d'un mètre d'épaisseur une accumulation de scories que ne vient troubler qu'un lit argilo-sableux ocre (qui sépare l'US 4 en deux strates). Ce liseré est ininterrompu sur la totalité de la surface ouverte. Sa faible puissance laisse penser qu'il s'agit 

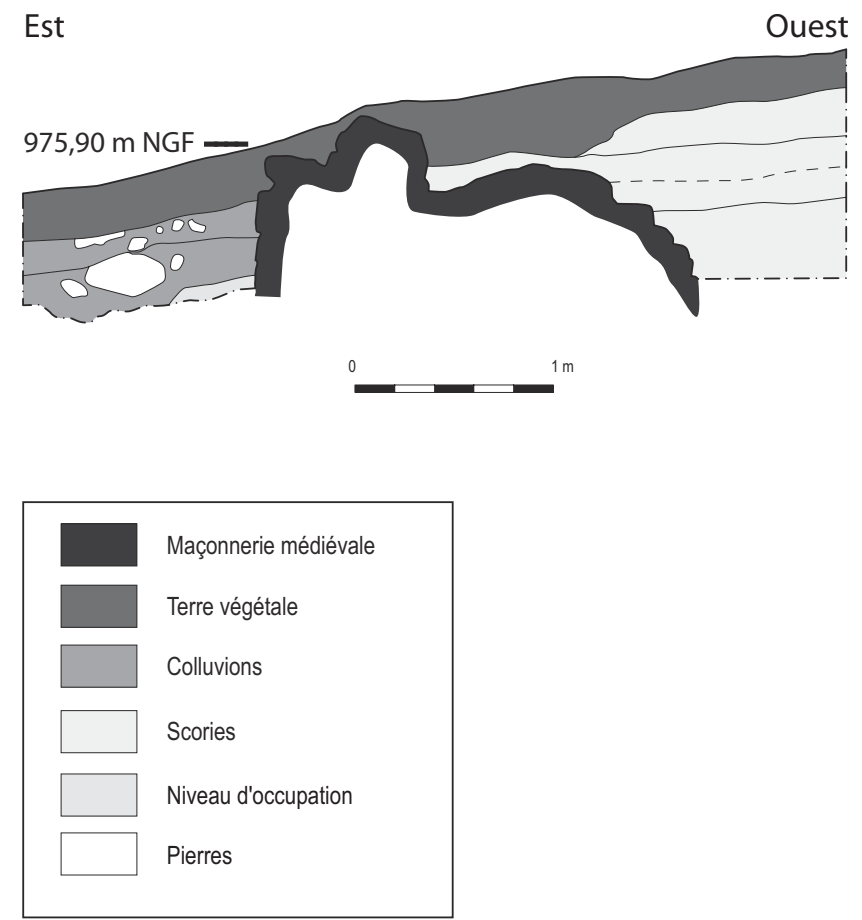

Figure 5 : Coupe du sondage archéologique. Figure 5: Archaeological section.

plus d'une phase d'arrêt relativement courte de l'activité sidérurgique que d'un recouvrement volontaire du ferrier. Il est à noter que l'étroitesse du sondage n'a pas permis d'atteindre les niveaux géologiques. En contrepartie, l'abondance de charbons de bois liés aux scories a facilité la réalisation d'une datation 14C. Les résultats ${ }^{3}$ de cette analyse donnent une fourchette chronologique comprise entre 1235 et 1405 avec une bonne probabilité entre 1235 et 1335 . Cette fourchette chronologique inscrit le ferrier dans les toutes premières phases, connues par les textes, d'échange de charbon contre du minerai entre le Couserans et la communauté de Sem (mine de Rancié) (Verna, 2001). Si l'on ajoute à cela que la couche datée est le niveau archéologique sommital de notre sondage, il est fort probable que l'activité sidérurgique sur ce site soit bien antérieure aux sources écrites qui nous sont parvenues.

3. Datation ARC 2438, Age ${ }^{14} \mathrm{C}$ conventionnel : $715+/-45$ BP (sigma 13C estimé de $-25,00$ pour mille vs PDB) Date $14 \mathrm{C}$ calibrée : $1220 \mathrm{cal}$ $\mathrm{AD}-1390$ cal AD (courbe de calibration «IntCal98 », Stuiver et al., 1998, Radiocarbon, 40). La datation ayant porté sur des branchettes présentant entre 20 et 30 cernes et le dernier cerne de croissance, il faut rajeunir la date ${ }^{14} \mathrm{C}$ calibrée de 15 ans environ pour obtenir la date d'abattage des bois. Cette date se situe donc entre 1235 et $1405 \mathrm{cal} \mathrm{AD} \mathrm{(à} \mathrm{95,4} \mathrm{\% ).}$

La probabilité que la date calibrée soit située :

- entre $1235 \mathrm{cal}$. AD et $1335 \mathrm{cal}$. AD est de 76,8 \%.

- entre $1335 \mathrm{cal}$. AD et $1405 \mathrm{cal}$. AD est de $21,7 \%$.
Autre information majeure liée à ce sondage, il existe un aménagement volontaire visant à circonscrire la masse des scories dans un espace défini au préalable. Ce muret se trouve en bord d'un chenal encore actif que les métallurgistes ont visiblement voulu protéger de leurs déchets afin d'éviter des curages répétitifs. Ce soin témoigne de l'importance que pouvait avoir le débit hydraulique de ce chenal. Dans le contexte pyrénéen et pour la période donnée, il est séduisant de penser à un usage de la force hydraulique pour la production sidérurgique (Verna, 2001).

A l'aide de ces données, une première estimation du cubage de ces amas de scories peut être avancée. En dessinant une forme ovoïde que fournit la prospection magnétique et en tenant compte de l'épaisseur moyenne constatée, le premier ferrier peut présenter un volume d'une centaine de $\mathrm{m}^{3}$. En revanche, le ferrier accroché sur les pentes du château ne dépasserait pas un volume de $60 \mathrm{~m}^{3}$. Le seul exemple de comparaison régional dont nous disposons à l'heure actuelle est le ferrier de Lercoul (distant d'une trentaine de kilomètres à vol d'oiseau). Son cubage a été estimé à $300 \mathrm{~m}^{3}$ (Dubois, 2000). Si l'on se réfere au classement proposé pour le site célèbre des Martys dans l'Aude, alors nos entités se classent dans la catégorie des petits ferriers (Decombeix et al., 2000). Évidemment ces éléments de comparaison sont issus du monde antique car nous ne pouvons que déplorer l'absence de données de ce type pour la période médiévale.

La différence de volume existant entre les deux ferriers ne repose pas, selon nous, sur une dégradation plus rapide $\mathrm{du}$ second ferrier posé sur une pente plus forte. En effet, nous n'avons constaté aucune anomalie topographie ni géophysique trahissant une accumulation particulière de scories en bas de pente pour le ferrier 2. Il y a bien ça et là des scories affleurantes ou remontées à l'occasion d'une taupinière, mais rien qui ne caractérise réellement un ferrier de bas de pente. Le volume estimé et la disposition au contact du château conduit à l'hypothèse suivante : si le ferrier 1 témoigne bien d'une activité de réduction du minerai de fer, le ferrier 2 devrait trouver sa raison d'être dans l'existence d'une forge. Sa présence dans le contexte d'un castrum doublé d'une activité minière n'aurait rien de surprenant. Bien au contraire, elle enrichit la problématique paléosidérurgique puisque nous serons amenés à travailler de la transformation du minerai jusqu'à l'objet.

\section{Conclusion}

Cette série de méthodes de prospection (microtopographie, prospections géophysiques, sondages pédologiques et sondage archéologique) nous a permis de localiser puis 
reconnaître dans les trois dimensions les vestiges d'une activité sidérurgique inattendue sur un site dont la fonction première reste la production de l'argent. À aucun moment il n'y a eu redondance entre les méthodes mises en œuvre, chacune ayant apporté son propre lot d'informations. Si cette phase prospective s'est étendue sur deux ans, il s'agit en premier lieu de contraintes logistiques. Les travaux futurs qui s'orientent vers une fouille de surface, sont correctement définis et l'importance du travail à accomplir a été proprement estimée. D'autre part, cette phase de prospection a permis d'entamer les recherches en terme de paléosidérurgie inscrivant cette thématique en amont de la fouille tout comme l'approche paléo-environnementale dont l'importance ressort à la vue des aménagements hydrauliques mis en ouvre par les hommes du Couserans. À n'en pas douter, le site de Castel-Minier offrira un éclairage nouveau sur l'extraction et la production de l'argent aux XIII' et XIV siècles. Plus étonnant, il devrait servir d'exemple et de base à une étude archéologique et archéométrique portant sur la période charnière dans les Pyrénées du passage du bas fourneau à la mouline.

\section{Remerciements}

Nous tenons à remercier le Service Régional de l'Archéologie Midi-Pyrénées ainsi que la commune d'Aulus-les-Bains pour leur soutien logistique qui a rendu possible l'exécution de ce programme. Remerciements également à Patrice Rieu qui nous a largement facilité l'accès à son terrain et montré combien il était sensible à l'histoire et au patrimoine de la vallée du Garbet.

\section{Bibliographie}

Beyrie, A., Florsch, N., Fluck, P., Leroy, M. et Tereygeol, F., 2005. La prospection géophysique en paléométallurgie, Dossiers Archéologie et Sciences des origines, 308, p. 74-79.

Blakely, R. J., 1996. Potential theory in gravity and magnetic applications, Cambridge, Cambridge University Press.

Dabas, M., Delétang, H., Ferdière, A., Jung, C. et W. HaioZimmermanN, W., 2006. La prospection, Paris, Errance, coll. "Archéologiques ".

Decombeix P.-M. et al., 2000. Réflexions sur l'organisation de la production du fer à l'époque romaine dans le bassin supérieur de la Dure, Gallia, 57, p. 23-36.

Dillmann Ph., Téreygeol F. et Verna C., à paraître. Premières analyses métallographiques des produits sidérurgiques trouvés sur le site de Castel-Minier, Revue d'archéométrie.

Dubois Cl., 2000. Lercoul (Pyrénées ariégeoises) : un site sidérurgique du $\mathrm{III}^{\mathrm{e}}$ siècle de notre ère, Gallia, 57, p. 53-62.

Panissod, C., Dabas, M., Hesse, A., Jolivet, A., Tabbagh, J. et TabBaGH, A, 1998. Recent developments in shallow-depth electrical and electrostatic prospectiong using mobile arrays, Geophysics, 63, 5, p. 1542-1550.

Scollar, I., Tabbagh, A., Hesse, A. et Herzog, 1990. Archaeological Prospecting and Remote Sensing, Edited by Arvidson, R. E., and Rycroft, M. J., Cambridge, Cambridge University Press.

Téreygeol F. (dir.), 2003. Le Castel-Minier (Aulus-les-Bains), Documents finaux de Synthèse, 90 p. et annexes.

Téreygeol F. (dir.), 2004. Le Castel-Minier (Aulus-les-Bains), Documents finaux de Synthèse, $68 \mathrm{p}$.

Verna C., 2001. Le temps des Moulines, fer, technique et société dans les Pyrénées centrales (XIII-XVI siècle), Paris, Publications de la Sorbonne. 